\title{
Efficient Spray-Coated Colloidal Quantum Dot Solar Cells
}

\author{
Kramer, I. J., Minor, J. C., Moreno-Bautista, G., Rollny, L., \\ Kanjanaboos, P., Kopilovic, D., Thon, S. M., Carey, G. H., Chou, \\ K. W., Zhitomirsky, D., Amassian, A. and Sargent, E. H.
}

Version Post-Print/Accepted Manuscript

Citation Kramer, I. J., Minor, J. C., Moreno-Bautista, G., Rollny, L., (published version) Kanjanaboos, P., Kopilovic, D., Thon, S. M., Carey, G. H., Chou, K. W., Zhitomirsky, D., Amassian, A. and Sargent, E. H. (2015), Efficient Spray-Coated Colloidal Quantum Dot Solar Cells. Adv. Mater., 27: 116121. doi:10.1002/adma.201403281
Publisher's Statement This is the peer reviewed version of the following article: Kramer, I. J., Minor, J. C., Moreno-Bautista, G., Rollny, L., Kanjanaboos, P., Kopilovic, D., Thon, S. M., Carey, G. H., Chou, K. W., Zhitomirsky, D., Amassian, A. and Sargent, E. H. (2015), Efficient Spray-Coated Colloidal Quantum Dot Solar Cells. Adv. Mater., 27: 116-121, which has been published in final form at https://dx.doi.org/10.1002/adma.201403281. This article may be used for non-commercial purposes in accordance with Wiley Terms and Conditions for Self-Archiving.

\section{How to cite TSpace items}

Always cite the published version, so the author(s) will receive recognition through services that track citation counts, e.g. Scopus. If you need to cite the page number of the TSpace version (original manuscript or accepted manuscript) because you cannot access the published version, then cite the TSpace version in addition to the published version using the permanent URI (handle) found on the record page. 
DOI: $10.1002 /(($ please add manuscript number $))$

Article type: Communication

\section{Efficient Spray-Coated Colloidal Quantum Dot Solar Cells}

Illan J. Kramer, James C. Minor, Gabriel Moreno-Bautista, Lisa Rollny, Pongsakorn Kanjanaboos, Damir Kopilovic, Susanna M. Thon, Graham H. Carey, Kang Wei Chou, Aram Amassian, Edward H. Sargent*

I. J. Kramer, J. C. Minor, G. Moreno-Bautista, L. Rollny, P. Kanjanaboos, D. Kopilovic, S. M. Thon, G. H. Carey, E. H. Sargent

Department of Electrical and Computer Engineering, University of Toronto, 10 King's College Road, Toronto, Ontario, M5S 3G4, Canada.

E-mail: ted.sargent@utoronto.ca

I. J. Kramer

IBM Canada Research \& Development Centre, 120 Bloor Street East, Toronto, Ontario, M4W 1B7, Canada.

S. M. Thon

Department of Electrical and Computer Engineering, Johns Hopkins University, 3400 North Charles Street, Baltimore, Maryland, 21218, USA

K. W. Chou, A. Amassian

Physical Sciences and Engineering Division, Solar and Photovoltaic Engineering Research Center (SPERC), King Abdullah University of Science and Technology (KAUST), Thuwal 23955-6900, Saudi Arabia

K. W. Chou

CELLS-ALBA Synchrotron, Carretera BP 1413, Km. 3.3, 08290 Cerdanyola del Vallès, Barcelona, Spain

I. J. Kramer and J. C. Minor contributed equally to this work

Keywords: photovoltaics, colloidal quantum dots

Solution-processed semiconductors offer the promise of low-cost, production-scale optoelectronic devices such as solar cells. Unfortunately, most reports are limited to lab-scale, batch-processing methods such as spin-coating and dip-coating. Strict requirements on their nanoscale and microscale morphology account for the sub-par performance of spray-coated bulk heterojunction organic and polycrystalline inorganic solar cells. Here we report recordefficiency spray-coated solar cells that employ an active layer that is deposited under ambient conditions and whose composition was selected to overcome the morphological constraints 
characteristic of other solution-processed technologies. Hypothesizing that planar solar cells based on pre-defined nanoparticles would provide a better platform for spray-coating, we explored the use of colloidal quantum dots (CQDs) as the active material. Only by developing a room-temperature spray-coating technique, and, by implementing a fully-automated process with monolayer control - an approach we term sprayLD - were we able to achieve solar cell performance, and a statistical distribution, superior to prior batch-processed methods along with hero performance of $8.1 \%$.

Scale-up manufacturing methods, in contrast with batch-level spin-coating onto discrete substrates, ${ }^{[1]-[3]}$ seek to coat large substrate areas using a continuous roll-to-roll process. Spray-coating (Figure 1a) has been used to deposit the active layer of photovoltaic devices based on organic materials ${ }^{[4]-[8]}$ and ternary nanocrystals $;{ }^{[9]}$ however, the power conversion efficiency $(\eta)$ of such devices has remained well below the performance of their batchprocessed counterparts. This reduction in performance upon scale-up is attributable to the failure of spray-coating to implement the nano- and micro-scale morphologies of organic (the interpenetrating nanoscale bulk heterojunction) and inorganic (well-passivated micron-scale polycrystalline) solar cells (Figure $1 \mathrm{~b}$, top \& middle). ${ }^{[8]-[11]}$

We turned our attention instead to CQDs, solution-processed semiconductors that have risen rapidly in performance in recent years as solar light harvesters. ${ }^{[12]}$ CQDs can in principle exceed the single-junction Shockley-Queisser solar efficiency limit ${ }^{[13]}$ through multi-junction $\operatorname{architectures}^{[14][15]}$ and multiple exciton generation. ${ }^{[16][17]}$ Since these materials are stored in and deposited from solution, they are well suited to large-scale, low-cost manufacturing processes. One recent report showed that a CQD-based ink is able to (with the aid of thermal annealing) produce a solar cell exhibiting just over $1 \%$ power conversion efficiency. ${ }^{[18]}$ Spraydeposited nanocrystals formed via in-flight crystallization/precipitation, ${ }^{[19][20]}$ or through a high temperature spray pyrolysis, ${ }^{[21]}$ have shown initial promise from a materials composition perspective, while mist-deposition of CQDs has been used in light emission applications. ${ }^{[22]}$ 
The promise of CQDs for spray-coated photovoltaics resides in significant part in the fact that, following their synthesis, the materials are already fully-formed - well-defined as nanocrystals - to serve as solution-phase precursors to CQD films. This notably simplifies the degree of morphological and chemical control required in the ensuing coating process (Figure $1 b$, bottom).

We developed a fully-automated spray-coater for CQD solar cell fabrication (Figure 2a, Figure SI1-1, Video SI-V1 and Video SI-V2). A fine mist containing oleic acid-capped CQDs dispersed in octane was atomized using pressurized nitrogen gas and deposited on a transparent conductive oxide/ $\mathrm{TiO}_{2}$ stack as described previously. ${ }^{[3][23]}$ Layers were treated using mercaptopropionic acid (MPA) in methanol to exchange the long aliphatic oleic acid ligand with the shorter MPA molecule, and were then rinsed with methanol. An air blade was used to apply a curtain of high pressure compressed dry air to aid in solvent drying. This process is repeated a number of times to achieve the final desired thickness (see Methods). This set-up is fully computer controlled to make fabrication consistent across multiple layers and multiple devices.

We first explored whether direct translation of existing spin-coating methods could produce devices having efficiency comparable to batch-processed reference samples. Unfortunately, spray coating CQDs resulted in the same dramatic reduction in performance characteristic of other solution-processed active materials. We investigated the origins of this performance loss, first inspecting film morphology using SEM and AFM (Figure SI2-1). These showed buckling and cracking of the films as well as an uneven, rough surface. For comparison, we studied our spin-cast controls (Figure 3a, top) and found that they also suffered morphological irregularities. We therefore investigated other limiting mechanisms, obtaining cross-sectional TEM (Figure 3b, left), which revealed striations in the materials stack. ${ }^{[24]-[26]}$ Using electron energy loss spectroscopy (EELS, Figure 3d) we found that the 
striations exhibited increased carbon content and a low level of CQD constituent elements such as sulfur and cadmium.

We posited that the accumulations of insulating organics could curtail electronic transport in the needed vertical direction, and we sought a new materials processing strategy to overcome these inhibitors to performance and consistency. An atomic layer deposition analogue, in which films would be spray-deposited and then developed monolayer-bymonolayer, could potentially overcome both the morphological and compositional limitations we had observed: the ligand-removing treatment would benefit from complete accessibility to the quantum dot layer to be exchanged, and volume-contraction-induced cracks would be readily in-filled using subsequent layers.

Devising such a new process would require greater control over the characteristics of the spray flux. We employed a very fine mist consisting of approximately $20 \mu \mathrm{m}$ diameter droplets. This facilitated more rapid and uniform evaporation of solvent as droplets impinged on the substrate surface. We also reduced the CQD concentration within the stock solution such that one $0.4 \mathrm{~s}$ spray pulse provided near-monolayer deposition of CQDs (Figure SI3-1).

This new approach, which we term sprayLD, led to locally smooth, crack-free films (Figure 3a, bottom and Figure SI4-1). We sought to investigate further whether a nondestructive surface-based probing method could provide diagnostic metrics of the film beneath. The elastic moduli of spin-cast films and sprayLD films were obtained using quantitative nanomechanical property mapping (see Methods). ${ }^{[27]} \mathrm{We}$ found that the elastic moduli of sprayLD films were more than one order of magnitude higher than those of spincast films (Figure 3c), indicating harder films. TEM images (Figure 3b, right) revealed no horizontal striations in sprayLD films. Further, the EELS of sprayLD films showed no thickness dependence on carbon, cadmium and sulphur in the film, indicating that no organicrich and CQD-poor layers were present within the CQD film. In sum, the sprayLD process had prevented residual organic material from accumulating in the film. The nanomechanical 
property mapping method offers the added benefit of investigating information related to depth profiles within hundred nanometer thick films, yet is a noninvasive surface-based probing technique. We employed grazing incidence small-angle X-ray scattering (GISAXS, Figure $3 \mathrm{e}-\mathrm{g}$ ) to investigate the origin of the harder sprayLD film. We found that the average particle spacing (Figure 3f) for spin-cast and spray-cast are $3.1 \pm 0.2 \mathrm{~nm}$ and $3.0 \pm 0.2 \mathrm{~nm}$; values that lie within uncertainty estimates of one another, but nevertheless indicate closer packing for sprayed films. The nearest neighbour GISAXS scattering peak is also found to be significantly sharper and narrower for sprayed films, particularly in the out-of-plane direction, where the spin-coated film scattering is the weakest (Figure 3g). These results point to a smaller interparticle spacing with narrower distribution of separations in the direction of charge transport and extraction. This improved packing density is believed to be due to the superior removal of organics from the CQD film via spray.

The photovoltaic performance of sprayLD devices under AM1.5 conditions is shown in Figure 4a. Devices exhibited solar power conversion efficiency of 8.1\%, a record for a solar cell employing a spray-coated active layer deposited under ambient conditions. The predicted short-circuit current density $\left(\mathrm{J}_{\mathrm{SC}}\right)$ of a sprayLD device calculated from an EQE spectrum (Figure 4b) matches the measured values under AM 1.5 conditions of approximately $24 \mathrm{~mA} / \mathrm{cm}^{2}$. In Figure 4c, histograms depict the measured distribution of performance of spincoated CQD devices vs. spray-coated CQD devices. Each data set includes all devices fabricated after the respective processing protocol (sprayLD vs. spin-coating) was finalized as a process of record. Each data point represents a device on a distinct substrate. The data are normalized to the area under the Gaussian fits. SprayLD devices have a higher mean $\eta$ than spin-coated devices (6.5\% and 5.2\% respectively; raw data can be found in Figure SI5-1) and a lower standard deviation $(0.7 \%$ and $1.4 \%$, respectively). The raw histogram of the unoptimized spray results can also be found in Figure SI5-1. A Welch's t-test was performed to determine if the difference between the two populations (spin and spray) was statistically 
significant. A p-value of 0.0001 was obtained, proving, with $99.99 \%$ confidence, that the sprayLD process yields statistically better performance than spin-coating ${ }^{[28]}$. Performance was also found to be uniform over a large area of $60 \mathrm{~cm}^{2}{ }^{[29]}$

In order to explain this improved performance, we measured the minority carrier diffusion length (Figure 5a) ${ }^{[30]}$ of charges in the sprayLD films compared with spin-coated films. The sprayLD materials achieved nearly $100 \mathrm{~nm}$ diffusion length, $25 \%$ greater than spincoated counterparts, suggesting that the defect density of sprayLD films was lower than that of spin films. Electroluminescence (EL) studies on devices indicated a low-energy luminescent state in spin-coated devices (Figure 5b, top) in the form of an infrared peak near $1600 \mathrm{~nm}$, far from the band edge at $1100 \mathrm{~nm}$. This signal was absent from the sprayLD device (Figure 5b, bottom). Prior studies have indicated that ligand configuration can contribute to long wavelength luminescence. ${ }^{[31]}$ Taken together, these results point to improved CQD film passivation and packing and therefore removal of an electronic defect leading to enhanced diffusion lengths via the sprayLD process.

This work features colloidal quantum dot solar cells fabricated using a roomtemperature spray-coating technique. It offers a route to scalable, roll-to-roll-compatible manufacturing process. Spray-coating was capable of producing films having excellent morphology and compositional purity via the introduction of an ALD-analogue process. The result was consistently higher average performance for the spray-coated devices, confirmed significant using statistical t-test. The champion sprayLD device achieved a power conversion efficiency of $8.1 \%$. More broadly, the work indicates that there exists no inherent compromise between manufacturability and performance.

\section{Experimental Section}

Synthesis of PbS CQDs and metal halide treatment: $\mathrm{PbS}$ quantum dots were synthesized according to a previously published method ${ }^{[32]}$. A solution-phase metal halide treatment 
$\left(\mathrm{CdCl}_{2}\right)$ was then carried out following a previously published method ${ }^{[23]}$. Specifically, the metal halide precursor ( $1 \mathrm{~mL}$ of $\left.\mathrm{CdCl}_{2}\right)$ and tetradecylphosphonic acid (TDPA) were dissolved in oleylamine with 13.6:1 Cd:TDPA molar ratio. This mixture was introduced into the CQD reaction flask after the sulfur source injection during the slow cooling process. A 6:1 $\mathrm{Pb}: \mathrm{Cd}$ molar ratio was adopted during the synthesis. At $30-35^{\circ} \mathrm{C}$, the nanocrystals were isolated by the addition of $60 \mathrm{~mL}$ of acetone then subjected to centrifugation. The nanocrystals were then purified by dispersion in toluene and re-precipitation with a mixture of acetone/methanol (1:1 volume ratio), then re-dissolved in anhydrous toluene. The solution was further washed with methanol two more times before finally dispersing it in octane at a concentration of $50 \mathrm{mg} / \mathrm{mL}$.

Substrate Preparation: Cleaned glass substrates coated with fluorine-doped tin oxide (FTO; Pilkington, TEC 15) were employed in this study. Two equivalent $\mathrm{TiO}_{2}$ electron accepting layers were found to be equal in performance and were used interchangeably in the process. The first used a sol-gel $\mathrm{TiO}_{2}$ mixture that was prepared, deposited and annealed according to a previously published recipe ${ }^{[33]}$. The second used a sputtered $\sim 50 \mathrm{~nm}$ layer of $\mathrm{TiO}_{2}$ (Kurt Lesker) using an argon pressure of 7.5 mTorr in an Angstrom Engineering A mod deposition system in an Innovative Technology glovebox and a deposition rate of $0.08 \AA$ /s. In either case, the substrates were then treated with a $120 \mathrm{mM} \mathrm{TiCl}_{4}$ solution at $70^{\circ} \mathrm{C}$ for $30 \mathrm{~min}$ followed by a rinse with deionized water and annealing step on a hot plate at $520^{\circ} \mathrm{C}$ for $45 \mathrm{~min}$ in air ambient. The samples were then stored in an $\mathrm{N}_{2}$-filled glovebox until just before device fabrication.

CQD Spray Deposition: The stock $50 \mathrm{mg} / \mathrm{mL}$ CQD in octane solution was diluted to 3.33 $\mathrm{mg} / \mathrm{mL}$ immediately prior to use. The total solution volume required for one device was 18.75 $\mathrm{mL}$, yielding a mass of oleic acid-capped CQDs of $62 \mathrm{mg}$. This solution was placed in a reservoir connected to the solution gravity-fed inlet of an Ikeuchi fine mist nozzle (BIMV8002S). The nozzle was pressurized to 45 psi using a $\mathrm{N}_{2}$ gas line. Another 45 psi $\mathrm{N}_{2}$ 
gas line provides activated piston control for the nozzle. Mercaptopropionic acid (MPA) was diluted in methanol $(\mathrm{MeOH})$ to $0.16 \%(\mathrm{v}: \mathrm{v})$ and placed in a reservoir for a Paasche VL airbrush pressurized with a $35 \mathrm{psi} \mathrm{N}_{2}$ gas line. A third $45 \mathrm{psi} \mathrm{N}_{2}$ gas line pressurized an additional Paasche VL airbrush or Ikeuchi flat-spray hydraulic nozzle (1/8(PT)VVP) loaded with $\mathrm{MeOH}$. Finally, a custom made air blade was connected to an 85 psi compressed dry air gas line. The setup can be seen in Figure SI1-1. Fabrication consisted of between 65 and 85 layers of a sprayed layer-by-layer procedure where each layer included:

1. $0.4 \mathrm{~s}$ actuated CQD nozzle followed by a $3 \mathrm{~s}$ pause

2. $1 \mathrm{~s}$ actuated MPA nozzle

3. $4 \mathrm{~s} \mathrm{MeOH}$ rinse for airbrush or $0.5 \mathrm{~s} \mathrm{MeOH}$ rinse for hydraulic nozzle

4. $40 \mathrm{~s}$ air blade drying

Two videos including the sprayLD procedure of the first three layers of a device and subsequent layers 4-75 are included in the supplementary information for reference at 1x time and sped up to $16 \mathrm{x}$ time, respectively.

The top contacts were deposited using an Angstrom Engineering Å mod deposition system in an Innovative Technology glovebox and consisted of $40 \mathrm{~nm}$ thermally evaporated $\mathrm{MoO}_{3}$ deposited at a rate of $1.0 \AA / \mathrm{s}$, followed by e-beam deposition of $50 \mathrm{~nm}$ of Au deposited at 1.5 $\AA / \mathrm{s}$, and finally $120 \mathrm{~nm}$ of thermally evaporated Ag deposited at $2.0 \AA / \mathrm{s}$.

Nanomechanical Properties Characterization by AFM: The AFM measurements were performed using PeakForce Quantitative Nanomechanical Property Mapping by Bruker®. Fast force curves were performed as the AFM scanned the samples' surfaces. The PeakForce QNM provides modulus data in addition to surface topology. Prior to measurement, the cantilever tip's radius and reflection sensitivity were measured via rough surface imaging and peak force measurement on quartz. In addition, the spring constant was measured via thermal vibration measurement. The surface indents for our samples were less than $1 \mathrm{~nm}$ using an 
indentation force of $5 \mathrm{nN}$. Only one cantilever was used and the samples were tested back to back to ensure comparability.

AM 1.5 Photovoltaic Performance Characterization: Current-voltage data were measured using a Keithley 2400 source meter. The solar spectrum at AM 1.5G was simulated to within class A specifications (less than $25 \%$ spectral mismatch) with a xenon lamp and filters (ScienceTech; measured intensity of $100 \mathrm{~mW} \cdot \mathrm{cm}^{-2}$ ). The source intensity was measured with a Melles-Griot broadband power meter through a circular $0.049 \mathrm{~cm}^{2}$ aperture. We used an aperture slightly smaller than the top electrode to avoid overestimating the photocurrent: the entire photon fluence passing through the aperture was counted as incident on the device for all analyses of $\mathrm{J}_{\mathrm{SC}}$ and $\mathrm{EQE}^{[34]}$. The spectral mismatch of the system was characterized using a calibrated reference solar cell (Newport). The total AM 1.5 spectral mismatch - taking into account the simulator spectrum and the spectral responsivities of the test cell, reference cell, and broadband power meter - was re-measured periodically and found to be $\sim 5 \%$. This multiplicative factor, $\mathrm{M}=0.95$, was applied to the current density values of the $\mathrm{J}-\mathrm{V}$ curve to most closely resemble true AM 1.5 performance ${ }^{[35]}$. The uncertainty of the current-voltage measurements was estimated to be $\pm 3.3 \%$.

EQE Measurements: External quantum efficiency measurements were obtained by applying chopped $(220 \mathrm{~Hz})$ monochromatic illumination $(450 \mathrm{~W}$ xenon lamp through a monochromator with order-sorting filters) collimated and co-focused with a 0.7 Sun intensity white-light source on the device of interest. The power was measured with calibrated Newport 818-UV and Newport 818-IR power meters. The response from the chopped signal was measured using a Stanford Research Systems current preamplifier feeding into a Stanford Research Systems lock-in amplifier set to voltage mode. The uncertainty in the EQE measurements was estimated to be $2.9 \%$.

Electroluminescence Measurements: Electroluminescence measurements were carried out by connecting a Keithley 2410 source meter to our devices and applying a range of forward bias 
voltages while reading the resultant current. The luminescence was collected through a set of lenses focused on an optical fiber and connected to an Ocean Optics NIR-512 spectrophotometer.

GISAXS Measurements: GISAXS measurements were performed on Beamline 06ID-1 (HXMA) of the Canadian Light Source. Monochromatic light was used with an energy of 7 $\mathrm{KeV}$. The marCCD SX-165 detector with a pixel size of $80 \mu \mathrm{m} \times 80 \mu \mathrm{m}$ and a total of 2048 $\times 2048$ pixels was used to record the scattering patterns. The images were dark-currentcorrected, distortion-corrected and flat-field-corrected by the acquisition software. Using a silver behenate powder standard, the sample-to-detector distance was determined to be 679 $\mathrm{mm}$. The angle of incidence of the X-ray beam was varied between 0.08 and 0.12 degrees, and an exposure time of 120 seconds was used. All films show primarily ring-like GISAXS patterns. We plotted azimuthally integrated intensity profiles and used Gaussian fitting plus an exponential background to determine the location of the scattering rings at $\mathrm{q} \approx 0.2 \AA^{\wedge}-1$. Conversion to real-space coordinates gave the average center-to-center nanocrystal spacings.

\section{Supporting Information}

Supporting Information is available from the Wiley Online Library or from the author.

\section{Acknowledgements}

Author 1 (IJK) and Author 2 (JCM) contributed equally to this work. The authors would like to Ikeuchi USA for useful discussions on the appropriate nozzles to use for our materials and solvents. This research is supported in part by IBM Canada Research and Development Center. This publication is based in part on work supported by Award KUS-11-009-21, made by King Abdullah University of Science and Technology (KAUST). The EM research described in this paper was performed at the Canadian Centre for Electron Microscopy, which is supported by NSERC and other government agencies. Research described in this paper was performed at the Canadian Light Source, which is funded by the Canada Foundation for Innovation, the Natural Sciences and Engineering Research Council of Canada, the National Research Council Canada, the Canadian Institutes of Health Research, the Government of Saskatchewan, Western Economic Diversification Canada, and the University of Saskatchewan. The authors would like to acknowledge the technical assistance and scientific guidance of C.Y. Kim. 


\section{References}

[1] Y. Liang, Z. Xu, J. Xia, S.-T. Tsai, Y. Wu, G. Li, C. Ray, L. Yu, Adv. Mater. 2010, 22, E135.

[2] Y. Wu, C. Wadia, W. Ma, B. Sadtler, A.P. Alivisatos, Nano Lett. 2008, 8, 2551.

[3] A.G. Pattantyus-Abraham, I.J. Kramer, A.R. Barkhouse, X. Wang, G. Konstantatos, R. Debnath, L. Levina, I. Raabe, M.K. Nazeeruddin, M. Grätzel, E.H. Sargent, ACS Nano $2010,4,3374$.

[4] G. Susanna, L. Salamandra, T.M. Brown, A. Di Carlo, F. Brunetti, A. Reale, Sol. Energy Mater. Sol. Cells 2011, 95, 1775.

[5] H.-Y. Park, K. Kim, D.Y. Kim, S.-K. Choi, S.M. Jo, S.-Y. Jang, J. Mater. Chem. 2011, $21,4457$.

[6] W. Nie, R.C. Coffin, J. Liu, Y. Li, E.D. Peterson, C.M. MacNeill, R.E. Noftle, D.L. Carroll, Appl. Phys. Lett. 2012, 100, 083301.

[7] T. Wang, N.W. Scarratt, H. Yi, A.D.F. Dunbar, A.J. Pearson, D.C. Watters, T.S. Glen, A.C. Brook, J. Kingsley, A.R. Buckley, M.W.A. Skoda, A.M. Donald, R.A.L. Jones, A. Iraqi, D.G. Lidzey, Adv. Energy Mater. 2013, 3, 505.

[8] P. Kumar, S. Kannappan, S. Ochiai, P.-K. Shin, J. Korean Phys. Soc. 2013, 62, 1169.

[9] V.A. Akhavan, B.W. Goodfellow, M.G. Panthani, D.K. Reid, D.J. Hellebusch, T. Adachi, B.A. Korgel, Energy Environ. Sci. 2010, 3, 1600.

[10] A. Goossens, J. Hofhuis, Nanotechnology 2008, 19, 424018.

[11] M.A. Majeed Khan, S. Kumar, M.S. AlSalhi, Mater. Res. Bull. 2013, 48, 4277.

[12] I.J. Kramer, E.H. Sargent, Chem. Rev. 2014, 114, 863.

[13] W. Shockley, H.J. Queisser, J. Appl. Phys. 1961, 32, 510.

[14] J.J. Choi, W.N. Wenger, R.S. Hoffman, Y.-F. Lim, J. Luria, J. Jasieniak, J.A. Marohn, T. Hanrath, Adv. Mater. 2011, 23, 3144.

[15] X. Wang, G.I. Koleilat, J. Tang, H. Liu, I.J. Kramer, R. Debnath, L. Brzozowski, D.A.R. Barkhouse, L. Levina, S. Hoogland, E.H. Sargent, Nat. Photonics 2011, 5, 480.

[16] J.B. Sambur, T. Novet, B.A. Parkinson, Science 2010, 330, 63.

[17] O.E. Semonin, J.M. Luther, S. Choi, H.-Y. Chen, J. Gao, A.J. Nozik, M.C. Beard, Science 2011, 334, 1530.

[18] M.P. Genovese, I.V. Lightcap, P.V. Kamat, ACS Nano 2012, 6, 865.

[19] L. Amirav, A. Amirav, E. Lifshitz, J. Phys. Chem. B 2005, 109, 9857.

[20] L. Amirav, E. Lifshitz, J. Phys. Chem. B 2006, 110, 20922.

[21] S.H. Im, Y.H. Lee, S.I. Seok, S.W. Kim, S.-W. Kim, Langmuir 2010, 26, 18576.

[22] T. Zhu, K. Shanmugasundaram, S.C. Price, J. Ruzyllo, F. Zhang, J. Xu, S.E. Mohney, Q. Zhang, A.Y. Wang, Appl. Phys. Lett. 2008, 92, 023111.

[23] A.H. Ip, S.M. Thon, S. Hoogland, O. Voznyy, D. Zhitomirsky, R. Debnath, L. Levina, L.R. Rollny, G.H. Carey, A. Fischer, K.W. Kemp, I.J. Kramer, Z. Ning, A.J. Labelle, K.W. Chou, A. Amassian, E.H. Sargent, Nat. Nanotechnol. 2012, 7, 577.

[24] I.J. Kramer, D. Zhitomirsky, J.D. Bass, P.M. Rice, T. Topuria, L. Krupp, S.M. Thon, A.H. Ip, R. Debnath, H.-C. Kim, E.H. Sargent, Adv. Mater. 2012, 24, 2315.

[25] D. Paz-Soldan, A. Lee, S.M. Thon, M.M. Adachi, H. Dong, P. Maraghechi, M. Yuan, A.J. Labelle, S. Hoogland, K. Liu, E. Kumacheva, E.H. Sargent, Nano Lett. 2013, 13, 1502.

[26] X. Lan, J. Bai, S. Masala, S.M. Thon, Y. Ren, I.J. Kramer, S. Hoogland, A. Simchi, G.I. Koleilat, D. Paz-Soldan, Z. Ning, A.J. Labelle, J.Y. Kim, G. Jabbour, E.H. Sargent, $A d v$. Mater. 2013, 25, 1769.

[27] D. Lee, S. Jia, S. Banerjee, J. Bevk, I.P. Herman, J.W. Kysar, Phys. Rev. Lett. 2007, 98, 026103.

[28] E.J. Luber, J.M. Buriak, ACS Nano 2013, 7, 4708. 
[29] I.J. Kramer, G. Moreno-Bautista, J.C. Minor, D. Kopilovic, E.H. Sargent, Appl. Phys. Lett. n.d.

[30] D. Zhitomirsky, O. Voznyy, S. Hoogland, E.H. Sargent, ACS Nano 2013, 7, 5282.

[31] C.A. Nelson, X.-Y. Zhu, J. Am. Chem. Soc. 2012, 134, 7592.

[32] M. a. Hines, G. d. Scholes, Adv. Mater. 2003, 15, 1844.

[33] H. Liu, J. Tang, I.J. Kramer, R. Debnath, G.I. Koleilat, X. Wang, A. Fisher, R. Li, L. Brzozowski, L. Levina, E.H. Sargent, Adv. Mater. 2011, 23, 3832.

[34] H.J. Snaith, Nat. Photonics 2012, 6, 337.

[35] J.M. Kroon, M.M. Wienk, W.J.H. Verhees, J.C. Hummelen, Thin Solid Films 2002, 403-404, 223. 
a

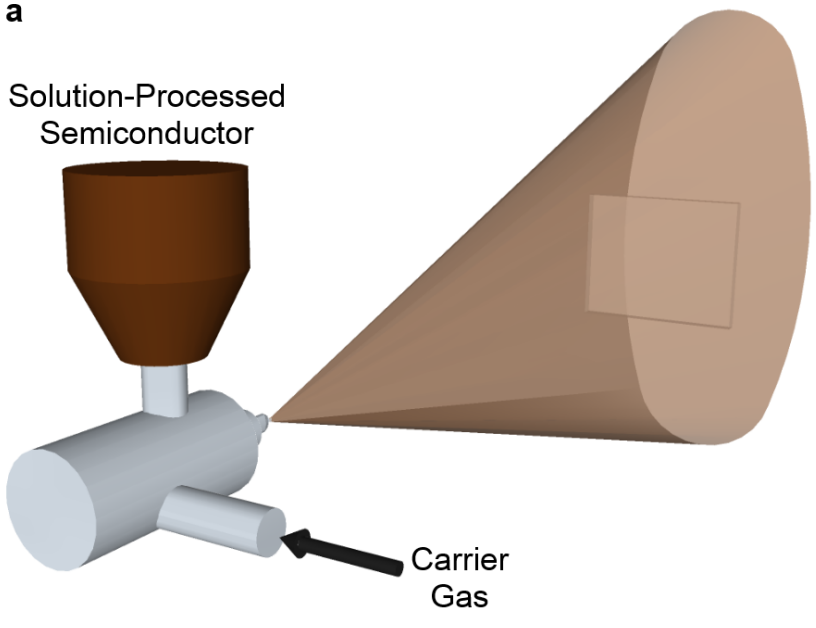

b

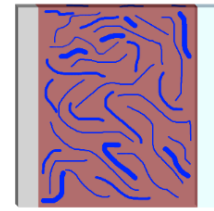

Organic

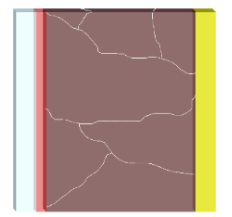

Multicrystalline

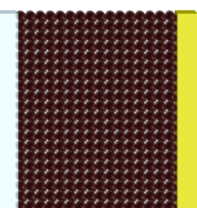

Colloidal Quantum Dot

Figure 1. (a) Sample mounted in the path of the elliptical spray cross section. (b) Illustrations of morphological considerations for (top) organic (middle) ternary multicrystalline and (bottom) CQD solar cells. The nanometer-scale interpenetrating morphology and micrometer scale domain sizes for the organic and multicrystalline devices, respectively, are difficult to achieve with spray-coating.

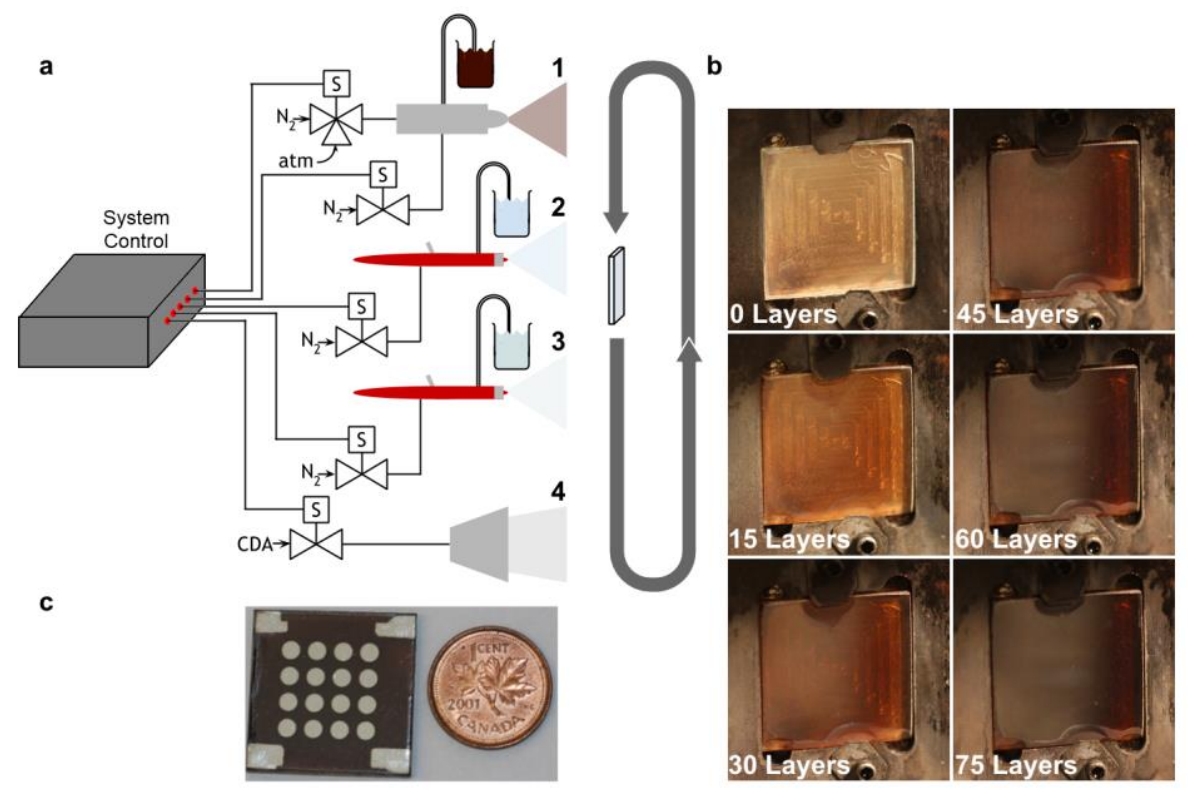

Figure 2. (a) Full setup of layer-by-layer spray deposition. Stage 1 involves the fine mist spraying of CQDs. Stages 2 and 3 use commercial air brushes to spray MPA diluted in methanol and pure methanol, respectively. In stage 4, an air blade applies a curtain of high pressure compressed dry air to aid in solvent drying. In all stages, two-way valves control carrier gas pressure to the nozzle, while in CQD deposition stage, a three-way valve controls the pilot gas to actuate spraying. We found that this enhanced control in the crucial CQD deposition phase enables uniform carrier gas pressure and therefore uniform CQD droplet size throughout the deposition cycle. All solenoid valves are controlled by a computer through a control printed circuit board. The looping of the sample through the 4 stages has been implemented as either a loop in space or in time. (b) A time-lapse series of photos of a square FTO-coated glass substrate as it is sprayed with the number of sprayLD layers. (c) A photograph of a sprayLD sample with sixteen $6.7 \mathrm{~mm}^{2}$ devices (apertured down to $4.9 \mathrm{~mm}^{2}$ ) on the same substrate. A one-cent coin is shown for dimension reference. 


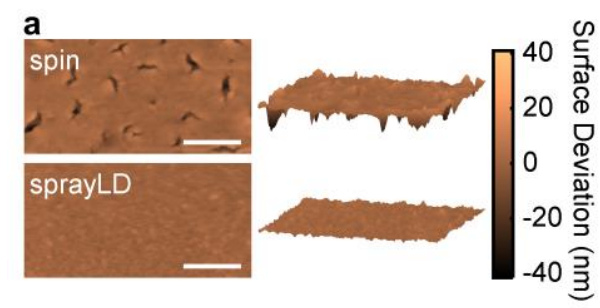

C
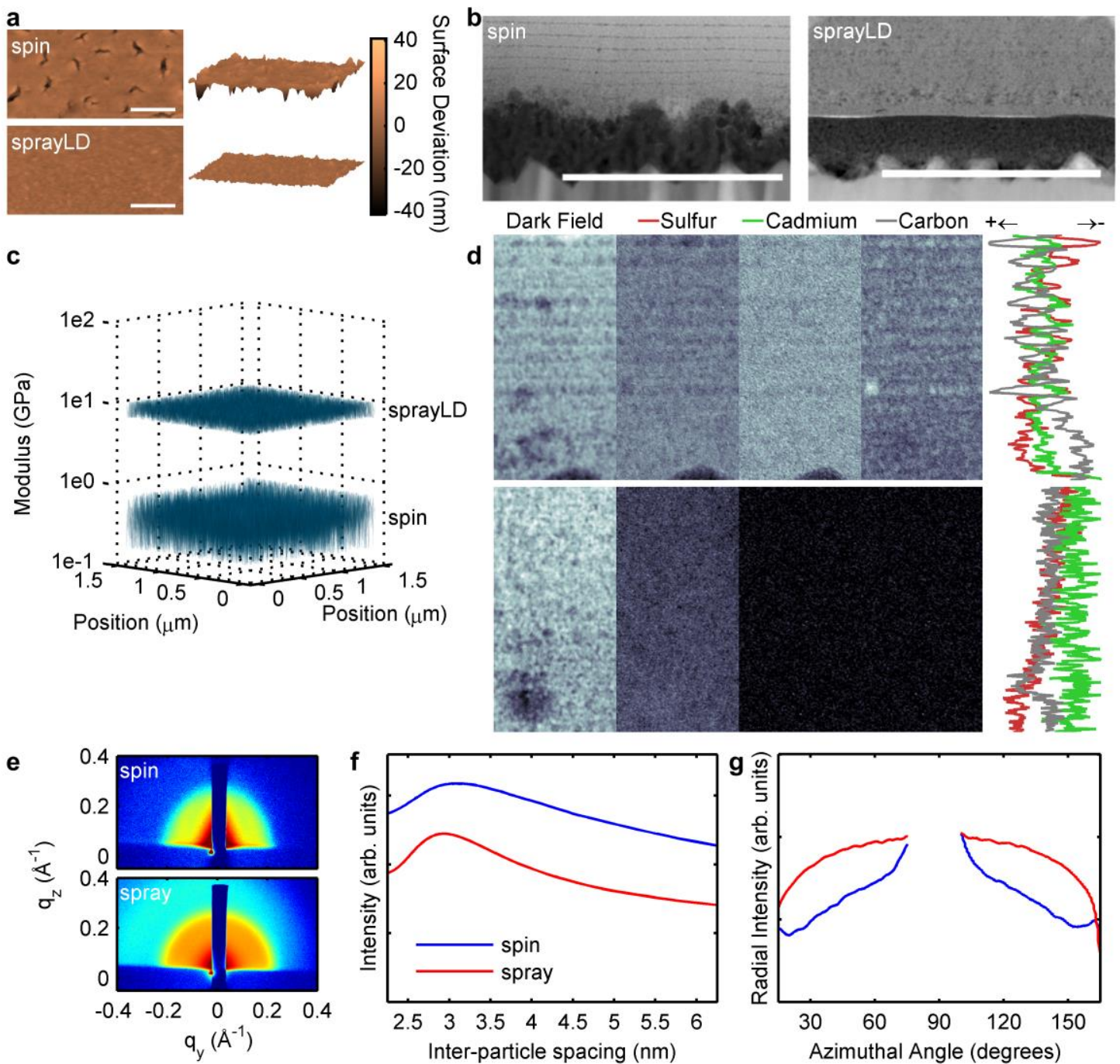

Figure 3. (a) Top and angled views of spin-cast films (top) and sprayLD films (bottom) obtained using AFM. Surface roughness (as the standard deviation of surface height) of spin and sprayLD samples is $2.0 \mathrm{~nm}$ and $1.8 \mathrm{~nm}$, respectively. (b) FIB-TEM of spin-coated and sprayLD films. The spin-coated film shows a clear striping effect indicating the presence of voids whereas the sprayLD film is stripe free. The white scale bars in (a) and (d) represent a distance of $500 \mathrm{~nm}$. (c) Elastic modulus of spin-cast and sprayLD films measured using AFM on a logarithmic scale. (d) EELS cross-sections of thick (top) and thin (bottom) CQD layers for the dark field, sulphur, cadmium and carbon along with the relative thickness variations of each (right graph). The sample with thick layers exhibits clear striping with the stripes being carbon rich and CQD-poor, while no such effects are visible for samples with near-monolayer control. (e) GISAXS plots of spin and unoptimized spray. Interparticle spacing (f) and radially integrated plots intensity (g) plots from (e). Even unoptimized, spray shows denser packing and better out-of-plane ordering than spin. 


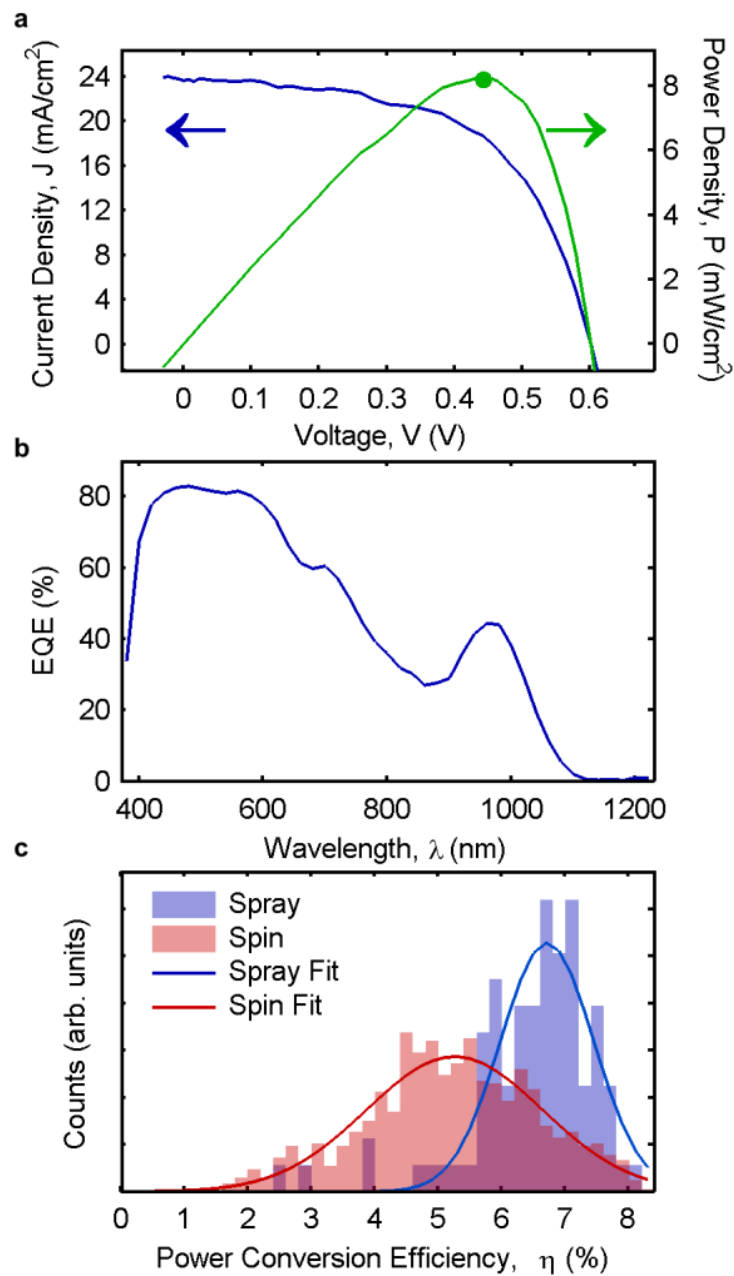

Figure 4. (a) J-V and P-V curves under AM 1.5 conditions of the best sprayLD device with static $\eta$ of $8.1 \%$ illustrated as a round marker. (b) EQE curve of sprayLD sample resulting in a predictive $\mathrm{J}_{\mathrm{SC}}$ of $\sim 23 \mathrm{~mA} / \mathrm{cm}^{2}$, matching the measured $\mathrm{J}_{\mathrm{SC}}$ under AM 1.5 conditions. (c) Histograms of device performance for spin and sprayLD devices with Gaussian fits overlaid. Data series were normalized to the Gaussian area to accentuate the higher mean value and narrower distribution of sprayed samples as compared with spin-coated ones. A Welch's t-test on the two populations resulted in a p-value of 0.0001 , indicating that the difference in performance is extremely statistically significant.
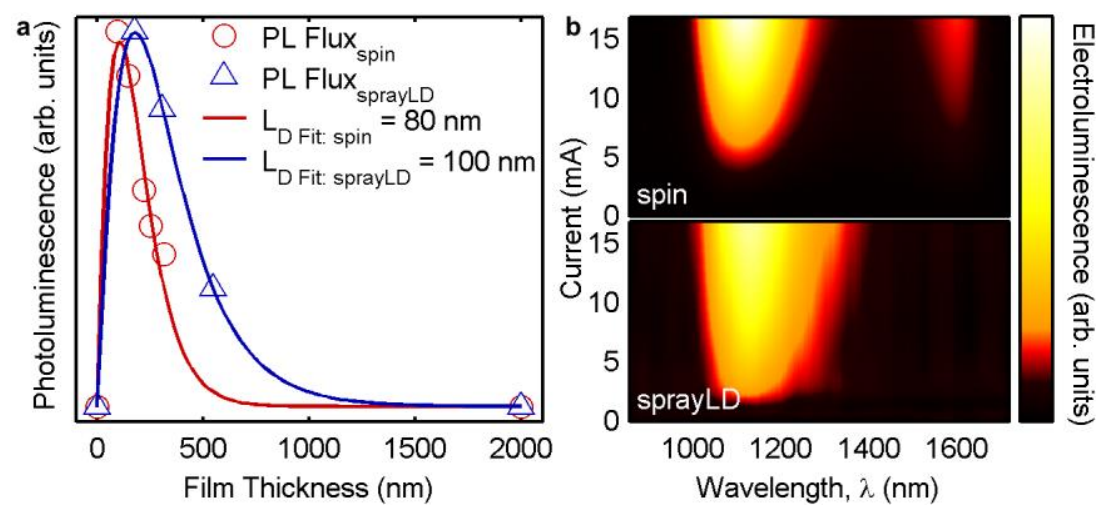

Figure 5. (a) Minority carrier diffusion length measurements of spin-coated and sprayLD films. The films exhibit $L_{D}$ of approximately $80 \mathrm{~nm}$ and $100 \mathrm{~nm}$, respectively according to the method in ref. ${ }^{[30]}$. (b) Electroluminescence measurements of spin (top) and sprayLD (bottom) films illustrating the presence of an electronic defect in the spin-coated film as manifested by a peak at $\sim 1600 \mathrm{~nm}$. The absence of the same peak in the sprayLD film implies the 
elimination of the electronic defect, thus explicating the enhanced diffusion length observed in (a).

A colloidal quantum dot solar cell is fabricated by spray-coating under ambient conditions. By developing a room-temperature spray-coating technique, and, by implementing a fullyautomated process with monolayer control - an approach we term sprayLD -we eliminate an electronic defect and achieve solar cell performance, and a statistical distribution, superior to prior batch-processed methods along with hero performance of $8.1 \%$.

Keyword: spray-coating, sprayLD, colloidal quantum dot, photovoltaic, nanocrystal

I. J. Kramer, J. C. Minor, G. Moreno-Bautista, L. Rollny, P. Kanjanaboos, D. Kopilovic, S. M. Thon, G. H. Carey, K. W. Chou, A. Amassian, E.H. Sargent*

\section{Efficient Spray-Coated Colloidal Quantum Dot Solar Cells}

ToC figure

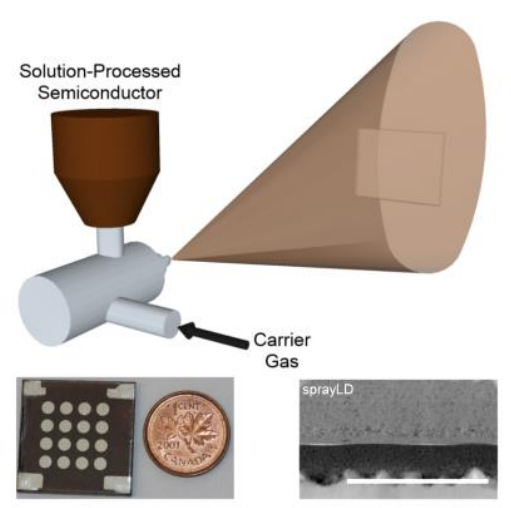

